\title{
Tools in ioChem-BD for Studying Chemical Reactivity: Nickel-Catalysed Borylation of C-F Bonds as a Case Study
}

\author{
Ana Mateo ${ }^{1}$, Moisés Álvarez ${ }^{1}$, and Carles Bo ${ }^{1}$ \\ ${ }^{1}$ CompChemCBo@ICIQ
}

May 5, 2020

\begin{abstract}
This manuscript reports a DFT based study on the reaction mechanism of the borylation reaction of aryl fluoride assisted by a nickel catalyst and a base, and the kinetic modelling of such process. The presence of the base modifies the classical cross-coupling reaction mechanism, by activating the diboron and promoting a boryl transfer reaction. Off-cycle reactions, such as some bis-phosphine/mono-phosphine equilibria showed large effects on the overall kinetics. Data publicly available within the ioChem-BD databases is integrated straightforwardly into the manuscript. We also show how the ioChem-BD Reaction Energy Profile Reports tool can be used for daily work and for accelerating catalyst discovery studies.
\end{abstract}

\section{Introduction}

Availability of computational research data in the form of databases following the FAIR principles (Findable, Accessible, Interoperable, Reusable) is playing a key role in accelerating catalysts discovery (Bo et al., 2018). ioChem-BD (Álvarez-Moreno et al., 2014), our computational chemistry results repository, fulfils such requirements, and it also provides tools for assisting in the quest of reaction mechanisms. In this interactive paper we are presenting those tools and how we used them in a case study: the Nickelcatalysed borylation or aryl-fluorides.

Aryl fluorides have the strongest C-heteroatom single bond in nature. The strength of the C-F bond arises from the small size and high electronegativity of the fluorine atom that makes fluorocarbons less reactive. This lack of reactivity (Shi, 2014) is the most relevant and characteristic chemical property of fluorocarbons. Remarkably aryl fluorides show an extraordinary metabolic activity, and this makes them especially attractive in the pharmaceutical industry (Wang et al., 2013). Replacement of hydrogen by fluorine in organic molecules can lead to useful pharmaceuticals and agricultural chemicals. The role of fluorine in medicinal chemistry has been reviewed recently,(Wang et al., 2013) and shows a wide variety of new drugs containing aryl fluorine or $-\mathrm{CF}_{3}$ groups. All these facts encouraged chemists to design catalytic C-F bond-forming, but also bond cleavage, protocols.

Very recently, Martin's(Liu et al., 2015) and Hosoya's(Niwa et al., 2015) groups reported an efficient C-F bond cleavage reaction via Ni-catalyst borylation, incited by the versatility and pivotal role of organoboron reagents (see Figure 1). Later, other groups presented different variations and tested different salts, bases, catalysts, ligands, and different diboron agents. (Zhou et al., 2016; Saito et al., 2016; Zhang et al., 2017; 
Niwa et al., 2017; Lee et al., 2016; Sakaguchi et al., 2017; Schwarzer et al., 2017; Lim et al., 2018) Borylation of C-X bonds is, therefore, a topic of current interest that makes a computational study attractive. There are plenty of open questions regarding the mechanism, the role of the base, and the reaction conditions.

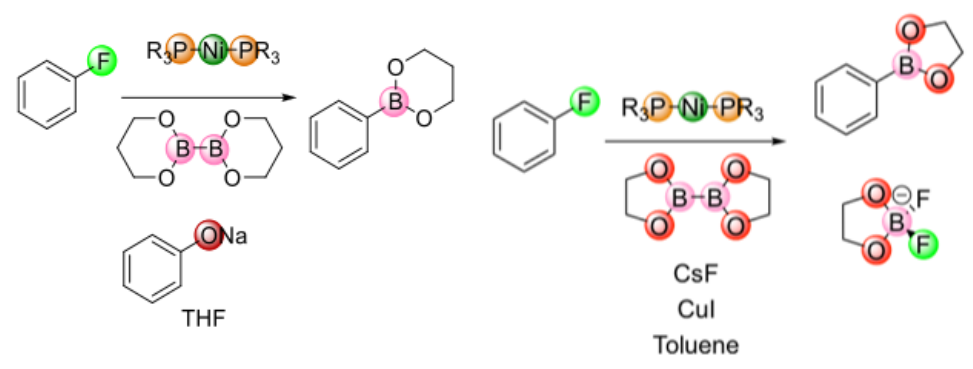

Figure 1: Scheme of the Martin (left) and Hosoya (right) reactions.

It was proposed that Martin's reaction follows a classical cross-coupling mechanism, as rational explanation for the products obtained. Cross-coupling reactions are processes in which two reactants, usually holding an activating group, react together with the aid of a metal catalyst. (Zapf, 2005) This mechanism comprises three elementary steps as shown in Figure 2: oxidative addition, in which one of the reactants react with the metal atom, which undergoes a formal 2-electron oxidation; transmetalation, where the second reactant exchanges with the activating group and adds itself to the catalyst; and finally in the reductive elimination the product is formed, and the catalyst recovered. Thus, the cross-coupling mechanism consists in the exchange of two fragments ( $\mathbf{X}$ and $\mathbf{Y}$ in Figure 2) between two different reactants $\left(\mathbf{R}-\mathbf{X}\right.$ and $\left.\mathbf{R}^{\prime}-\mathbf{Y}\right)$ assisted by a metal complex $\mathbf{M L}_{2}$ as catalyst.

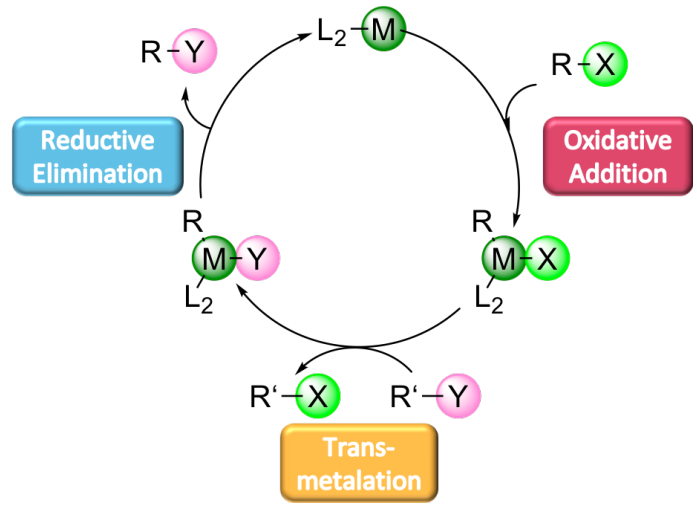

Figure 2: Classical Cross-Coupling reaction mechanism.

However, what surprises the most in the reactions in Figure 1 is the presence of a base, such is sodium phenolate or $\mathrm{CsF}$, which a priory has any role in the mechanism but its presence is crucial. Thus, the participation of the diborane reagent and a base made us to think in the possibility of a more complex mechanism in which the base somehow participates.

Herein we present results of a computational study aimed at shedding light into the mechanism of Martin's reaction (Liu et al., 2015) using Density Functional Theory (DFT) based methods. This interactive 
manuscript includes live 3D molecular structures, editable 2D graphs, a python notebook, and it showcases some tools in ioChem-BD (Álvarez-Moreno et al., 2014) that facilitate studying chemical reactivity. Moreover, we show how the results of computational chemistry studies, as elaborated by these tools, can then be embedded in a manuscript like this one, and published straightforwardly.

\section{Computational Details}

All the calculations in this paper were carried out using Gaussian09 program package (Frisch et al.) revision D.01. Several Density Functional Theory (DFT) based methods were used: the range separated functional wB97X-D (Chai and Head-Gordon, 2008) was used as the primary method. All the geometry optimizations, harmonic vibrational frequencies, and energies were calculated using the $6-311 \mathrm{G}^{* *}$ basis set(Schuchardt et al., 2007) for C, H, B, O, F and N atoms; for Ni and Cs atoms we used the LANL2DZ basis set (Schuchardt et al., 2007), which includes the corresponding effective core potentials. The SMD (Marenich et al., 2009) solvent model was chosen to include THF solvent effects implicitly. Gibbs free energy calculations included entropy corrections as introduced by Martin.(Martin et al., 1998) A model ligand, trimethylphosphine $\left(\mathrm{PMe}_{3}\right)$, was considered instead of the bulkier tricyclohexylphosphine $\left(\mathrm{PCy}_{3}\right)$ used in the experiments. A data set collection of all computational results is available in the ioChem- BD repository (Álvarez-Moreno et al., 2014) and can be accessed by following this link.

\section{Results and Discussion}

The optimal geometries and relative energies of all possible intermediates and transition states (TSs) were evaluated by means of DFT based methods. In Martin's reaction, the $\mathrm{Ni}\left(\mathrm{PCy}_{3}\right)_{2}$ complex plays the role of catalyst and the two reactants that exchange fragments are aryl fluoride and the diboron 5,5-dimethyl-1,3,2dioxaborolane $\left(\mathrm{B}_{2}\right.$ nep $\left._{2}\right)$. Therefore, there is any reason preventing this reaction to follow a classical crosscoupling mechanism. Also, the oxidative addition and the reductive elimination as the first and last steps, respectively, seem to be well accepted. However, as mention above, the recipe contains sodium phenolate, which its role is unraveled yet. In order to understand the basics of the reaction mechanism, we outlined three possible scenarios for the transmetalation step: (a) absence of base, (b) presence of the phenolate ion, (c) participation of both the phenolate ion together with its $\mathrm{Na}^{+}$counter-ion. Also, considering the labile character of the metal-phosphine bonds,(Taube, 1952) we examined the reactivity of both mono- and bis-phosphine complexes in each reaction step.

\section{Reaction Mechanism}

We characterized reactants, products, intermediates, and transition states (TS) of all possible mechanisms for the Martin's reaction, and constructed the reaction energy profile (REP) for each one. This is for both mono- and bis-phosphine complexes three pathways (classical transmetallation, with $\mathrm{PhO}^{-}$, and with $\mathrm{NaPhO}$ ). The six REPs were plotted together in Figure 3. It permits seeing the differences between the reaction pathways in global manner.

Detailed analysis of REPs revealed the lowest reaction energy path, which it is schematically shown in Figure 4. The molecular structures of all species in Figure 4 as well as the corresponding transition states can be found in Figure 5, which shows the 3D models interactively. The HTML code needed to embed the structures into this article is easily generated by ioChem-BD with a simple one-click process. For each structure we also provide a link for accessing the corresponding entry live in the ioChem-BD databases. For each entry, input files, optimized geometries, energies, vibrational frequencies, and other molecular properties are available. 


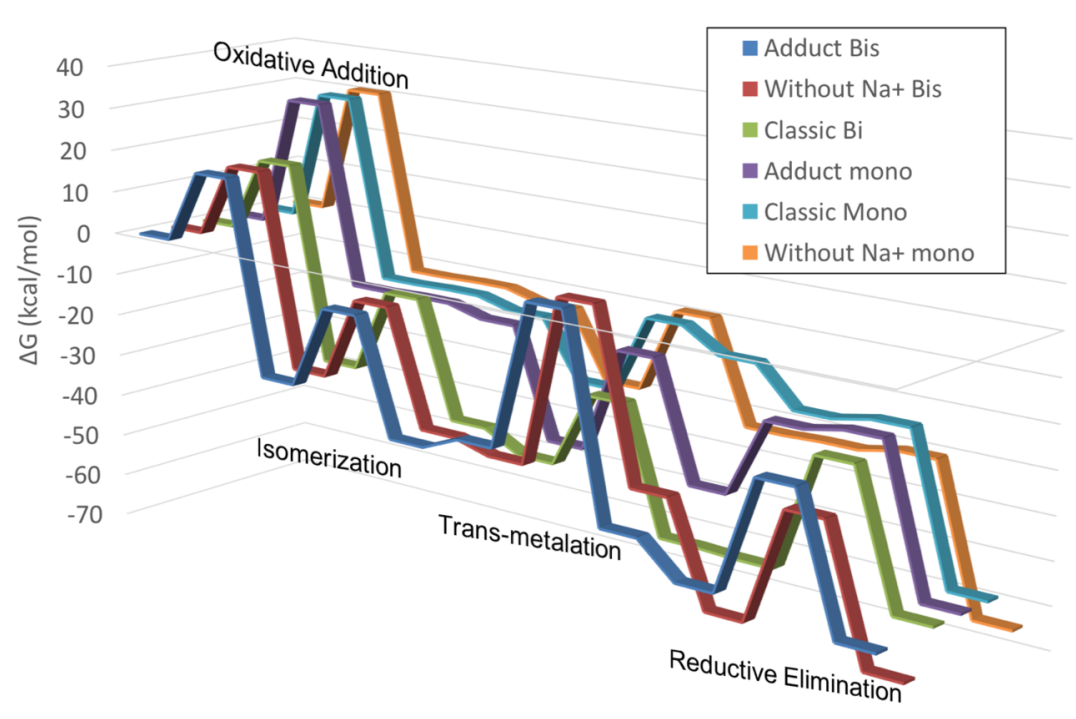

Figure 3: $\quad$ Reaction energy profiles of all possible mechanisms for the Martin's reaction.

Moreover, the ioChem-BD tool called Reaction Energy Profile Reports enables building REPs directly from the uploaded data, and then to export the graph. We selected the species involved in the lowest energy reaction path, built the REP, dowload the corresponding plot.ly file, and then embed it in this paper, directly without any other manipulation. The result of this straightforward process is Figure 6.

After forming the catalyst $\mathrm{Ni}\left(\mathrm{PMe}_{3}\right)_{2}$ ( $\boldsymbol{B}$ ) from the precursor $\mathrm{Ni}(\mathrm{COD})_{2}(\boldsymbol{X})$ and $\mathrm{PMe}_{3}(\boldsymbol{Z})$, the oxidative addition takes place leading to $\boldsymbol{E}$. The next step is the isomerization of intermediate $\boldsymbol{E}$ obtained in the oxidative addition reaction step. The square-planar complex $\boldsymbol{E}$ experiments a cis/trans isomerization process regarding the mutual disposition of the two phosphine ligands. This step is energetically less costly in monophosphine complexes $\boldsymbol{E} \boldsymbol{M}$ and $\boldsymbol{F} \boldsymbol{M}$. Note that species $\boldsymbol{E}$ could not accommodate the diboron reagent because steric congestion.

The next step is the transmetalation. In the present case, the transmetalation consists in the reaction between the intermediate obtained after the isomerization $\boldsymbol{F M}$ and the diboron $(\boldsymbol{C})$. In this step, both the Ni-F bond and the diboron B-B bond break, the boryl boron moiety will end coordinated to the metal centre, and the other boron moiety is eliminated together with the fluorine ligand. The participation of one molecule of the neutral base, $\mathrm{NaOPh}$, provided the most favourable reaction pathway. Therefore, the mono-phosphine trans intermediate $(\mathbf{F M})$ reacts with the adduct $\mathbf{G}$ forming the pre-adduct $\mathbf{G M}$. Then, the transmetalation goes through the TSGM-HM giving the HM post-adduct. This HM post-adduct reacts with phosphine $\mathbf{Z}$ giving the third intermediate $\mathbf{I}$ and a sub-product $\mathbf{J}$. Therefore, the most favourable mechanism is a non-classical transmetalation, with the participation of one molecule of the base in neutral form $\mathrm{NaOPh}$.

Finally, we reach the last step of the reaction mechanism, in which the new C-B bond is formed and the catalyst recovered by formal reduction of the metal atom. I undergoes the reductive elimination and releases the product.

ioChem-BD Reaction Energy Profile Reports is a tool designed to facilitate discovery of reaction mechanisms. After a successful run on a given molecular structure, the results are uploaded into ioChem-BD. Then, this 


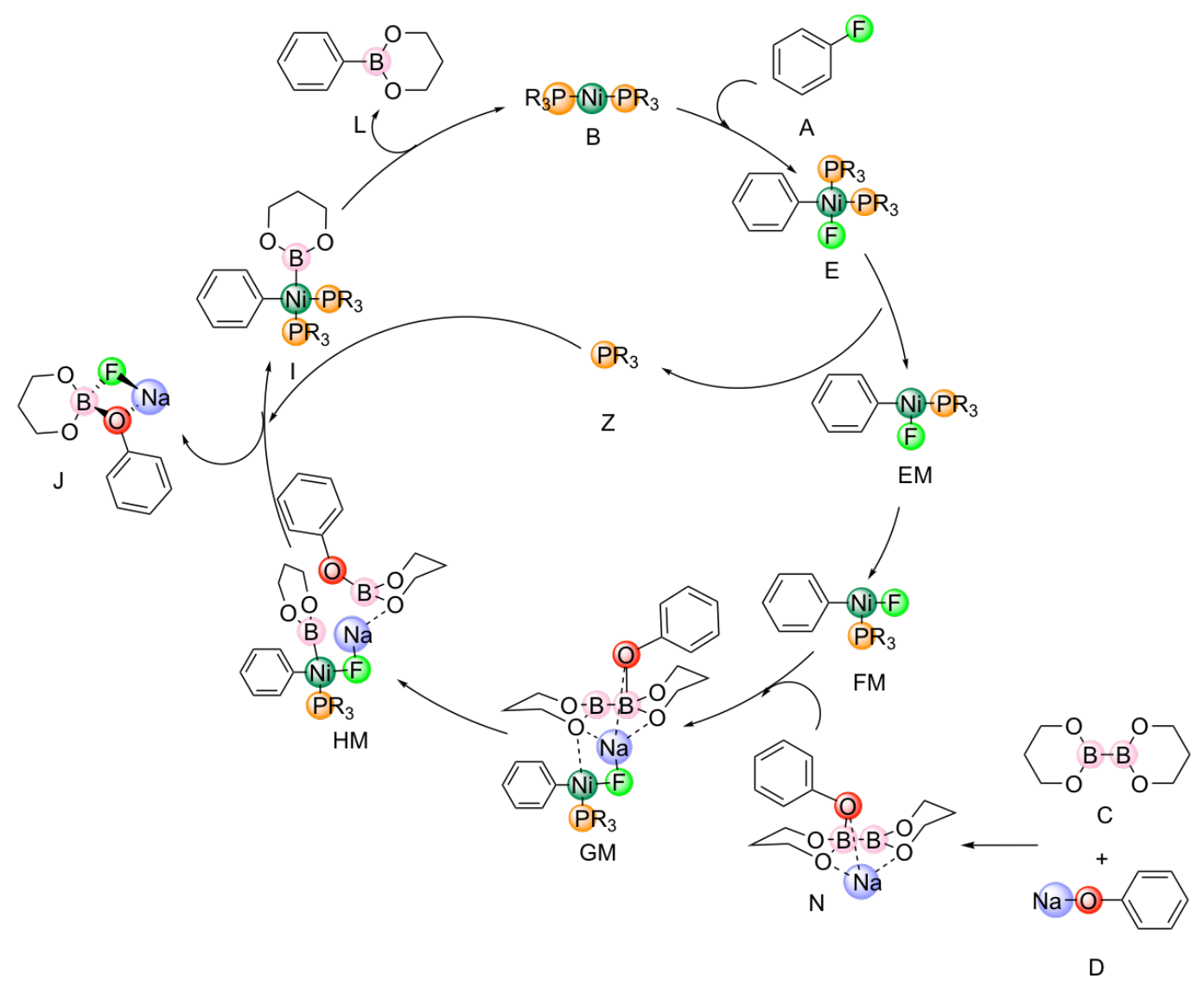

Figure 4: Proposed mechanism for the Martin's reaction.

tool enables selecting a set of entries, so all those species participating in the mechanism, and then to add reaction steps, the corresponding mathematical formulas needed for calculating the energy of each reaction step properly, and also to label each step. Figure 7 depicts a screenshot of the form used to define all that information. Several Series can be defined as well as what we call Variables, actually constant values that make writing the mathematical expressions even easier. Note in Figure 7 that R1 is defined as the sum of the reactants $(\mathrm{c} 3+\mathrm{c} 4)$, and that simple algebraic expressions are admitted.

When all the information is entered, so reaction steps and corresponding formulas defined, users can choose which kind of energy to plot (Potential Energy, Free Energy, Enthalpy,..), in which units (kcal/mol, kJ/mol, $\mathrm{eV}$ ), and get the plot in one-click. Figure 6 is the direct result of this operation. ioChem-BD uses plot.ly libraries for constructing the graph, so this makes very easy to edit the plot details, and also to publish it as is. 


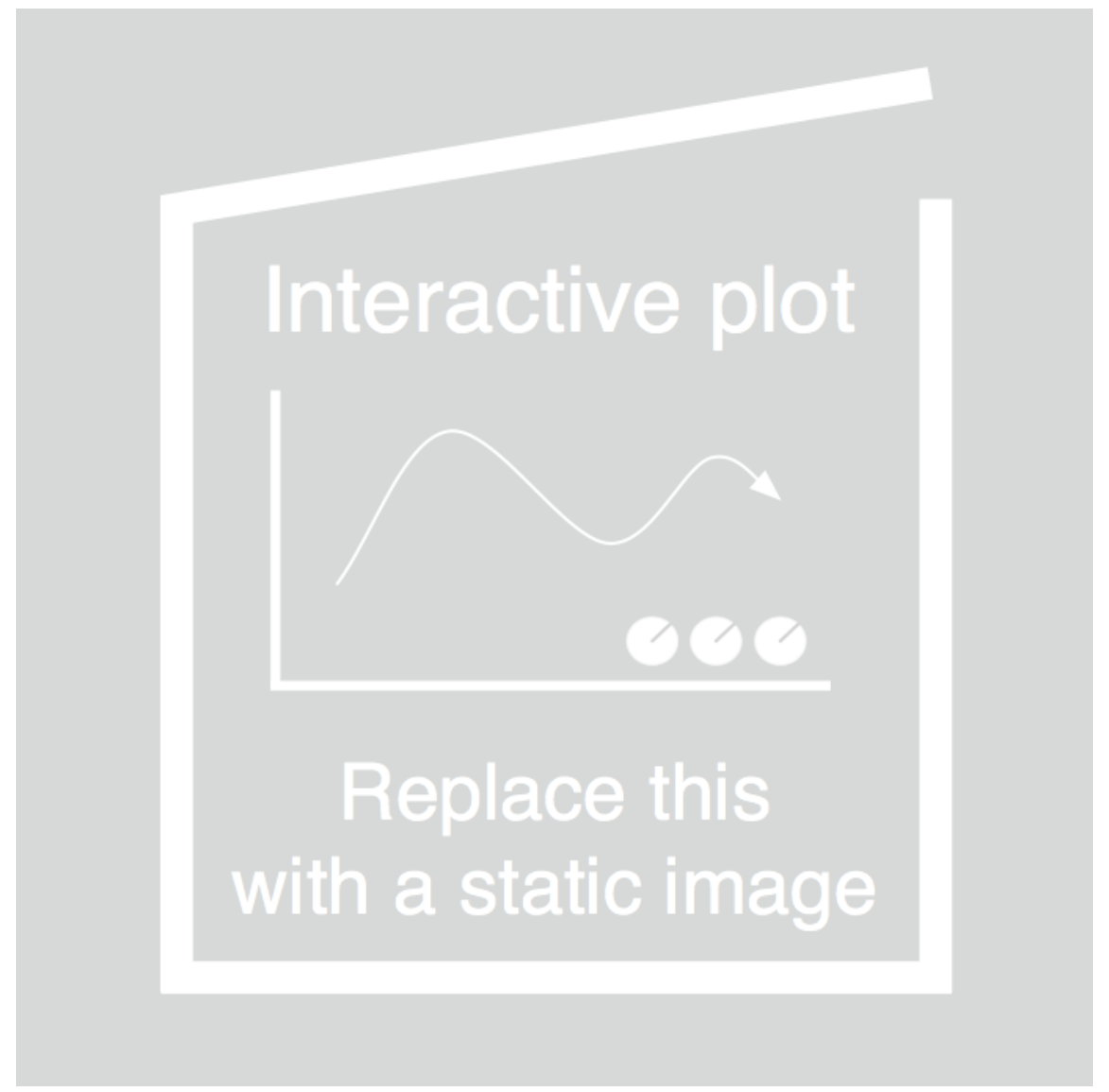

Figure 5: Molecular models for reactants, intermediates, transition states and products that participate in the most favourable mechanism of Martin's reaction. The link points to the original data in ioChem-BD databases.

\section{Kinetic Analysis of the reaction}

In Computational Homogeneous Catalysis, the classical way of studying a reaction is through the characterization of reactants, products, all possible intermediates, and the corresponding Transition States (TS) for each single chemical transformation, so for each reaction step. Connections between species define the reaction mechanism, or in other words, the reaction map. As it was exemplified by Besora et al. (Besora and Maseras, 2018) in a recent review, further kinetic modeling provides additional insight and understanding. After a reaction mechanism has been proposed, running a kinetic analysis could be considered as a stress-test for the mechanism. The Eyring equation (Eyring, 1935) permits to obtain the kinetic constant of each single reaction step, which can be calculated easily from the activation energies.

As a first try, we considered all the elemental reaction steps of the most favourable mechanism, this is the nine reactions depicted in Figure 8. This includes nine transformations: 


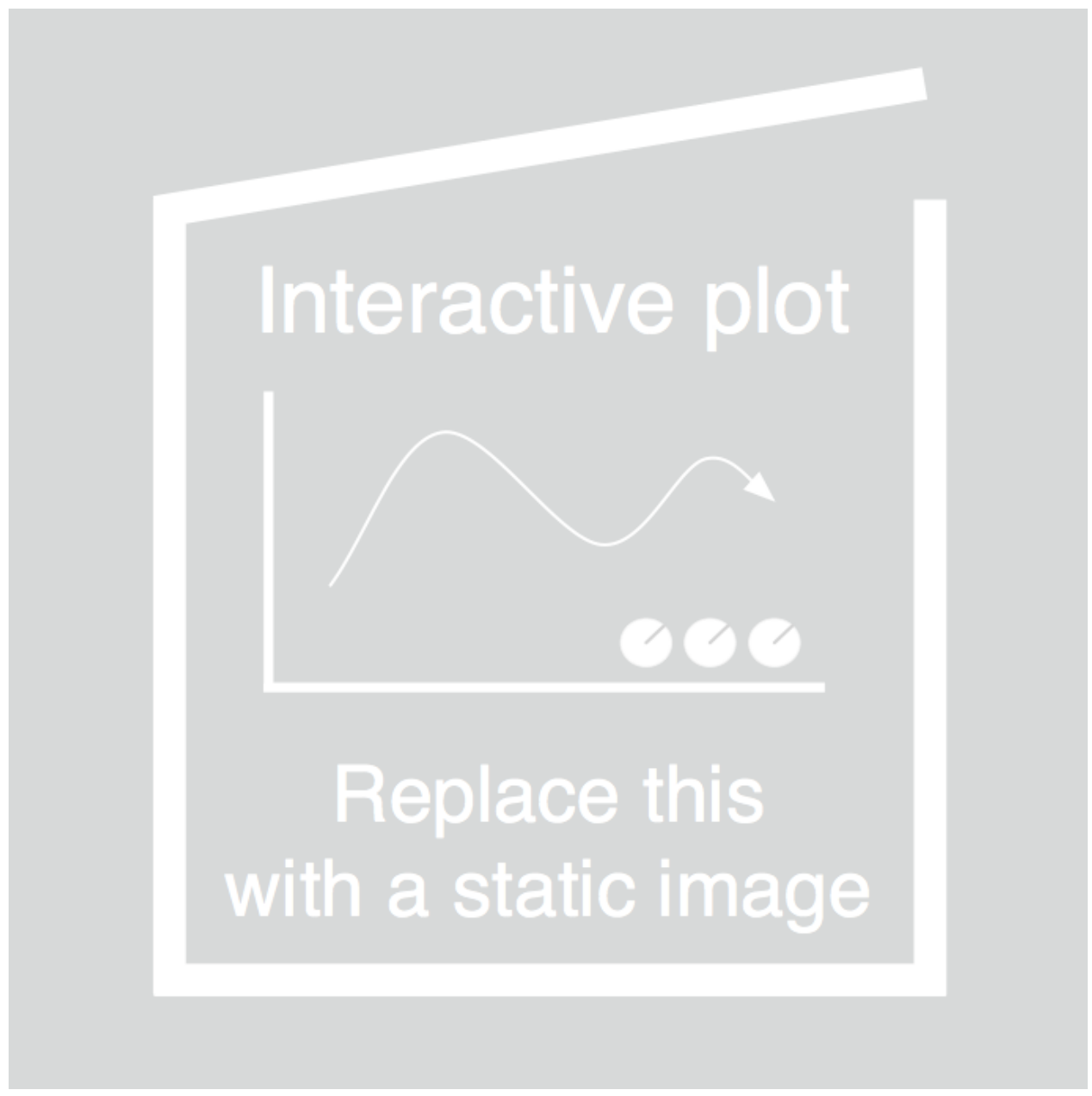

Figure 6: Reaction Energy Profile for the most favourable reaction mechanism of Martin's reaction, as generated by ioChem-BD Reports tool.

1. The initial form of the catalyst $\mathrm{Ni}(\mathrm{COD})_{2}$ evolves to $\mathrm{Ni}\left(\mathrm{PR}_{3}\right)_{2}$, the catalytically competent species.

2. Oxidative Addition, where the catalyst reacts with the aryl fluorine.

3. Dephosphination

4. Isomerization of the mono-phosphine complex.

5. Sodium phenolate activates the diboron compound forming an adduct.

6. Formation of a supramolecular complex between the diboron adduct and the trans monophosphine complex.

7. Transmetalation, where a boron takes the place of the fluorine ligand.

8. In this step we obtain the first product, with a tetrahedral boron, and the second monophosphine intermediate.

9. Reductive elimination step, where the desired product is obtained and the catalyst recovered.

Taking into account the same initial concentrations for reactants and catalyst used in the experiments, that were: Aryl fluoride $[\mathbf{A}]=0.25 \mathrm{~mol} \cdot \mathrm{dm}^{-3}$, Phosphine $[\mathbf{Z}]=0.05 \mathrm{~mol} \cdot \mathrm{dm}^{-3}, \mathrm{Ni}(\mathrm{COD})_{2}[\mathbf{Y}]=0.0125 \mathrm{~mol} \cdot \mathrm{dm}^{-3}$, diboron $[\mathbf{C}]=0.75 \mathrm{~mol} \cdot \mathrm{dm}^{-3}$, base $[\mathbf{D}]: 0.75 \mathrm{~mol} \cdot \mathrm{dm}^{-3}$, the system of kinetic equations was solved and the concentration vs time profile obtained plotted and included in Figure 9 as a python notebook. 


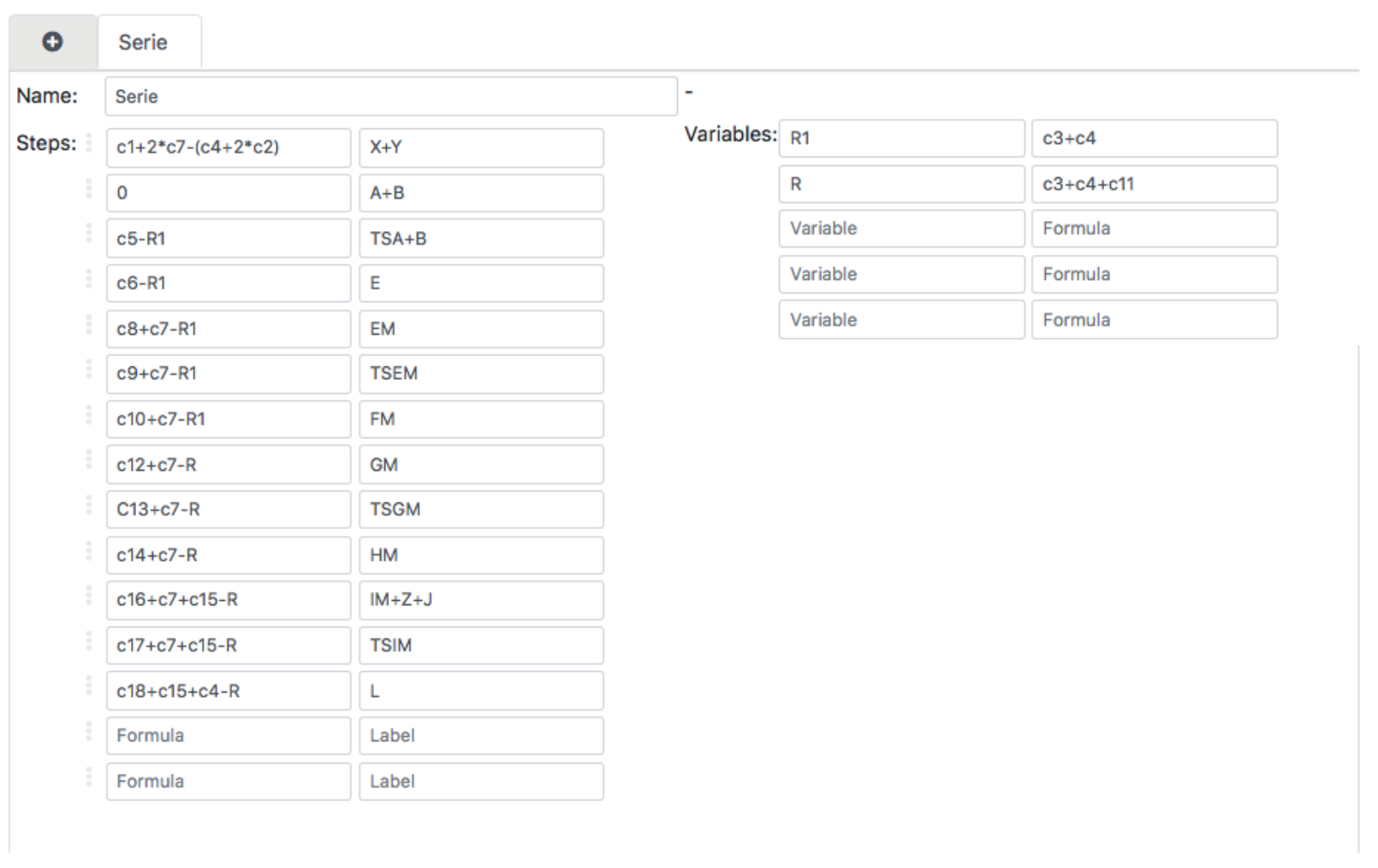

Figure 7: Reaction Energy Profile Report Screenshot .

According to the experimental reports, the reaction provided $80 \%$ of the product within 12 hours. (Liu et al., 2015) This is thus the target that we aimed our mechanism would fulfil. In Figure 9 we present the result of solving the system of kinetic equations described above in Figure 8, so concentration versus time profiles for all the species under the nine kinetic equations regime. By analysing this data, the total time required to reach $80 \%$ conversion is as short as 8000 seconds. This result is by far to be close to the reaction time reported experimentally. This raises issues about the accuracy of our calculations, or about the simplicity of the nine-steps mechanism kinetic model.

Actually, there is a bunch of elemental reactions that involve species participating in the most favourable mechanism and that they were not included in the kinetic model. At least 21 additional reactions, as collected in Figure 10, might play a role. Just by adding reactions labelled 11, 12 and 14 to the kinetic model, this is those involving bis-phosphine/mono-phosphine equilibria, the computed $80 \%$ conversion time increased from $8000 \mathrm{~s}(2.2 \mathrm{~h})$ till $64000 \mathrm{~s}(17.8 \mathrm{~h})$, much closer to the experimental value. That could be explained since the first cis/trans isomerization barrier is higher in the bis-phosphine complex than in the mono-phosphine because the bis-phosphine compounds are more stable than the mono-phosphine species. Additionally, since phosphine is in high concentration, in excess actually, favours the formation of bis-phosphine compounds, and consequently, the reaction becomes slower because dephosphination

has a high barrier to go from stable to a less stable species. 


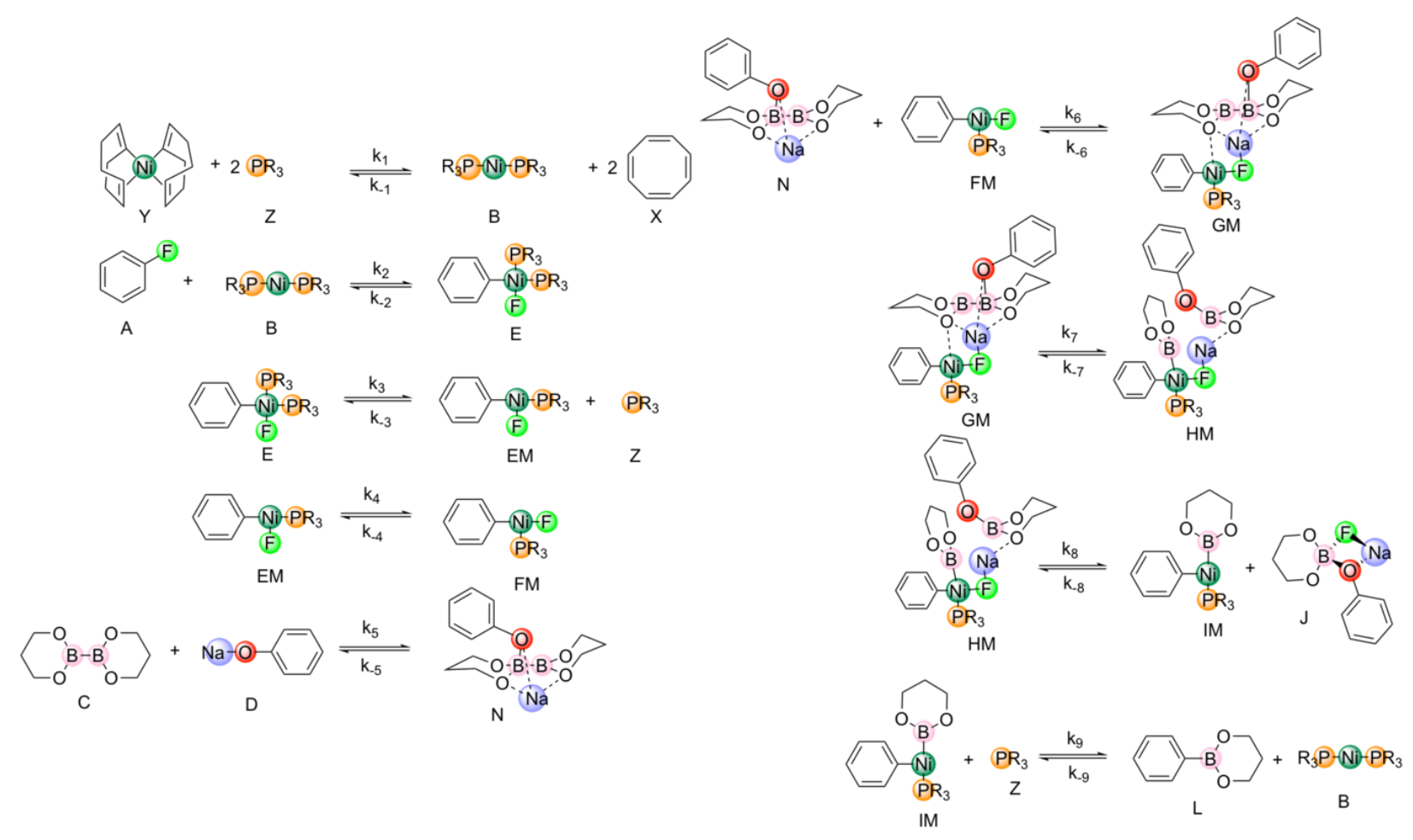

Figure 8: Elemental Reactions of the most favorable mechanism of Martin's reaction

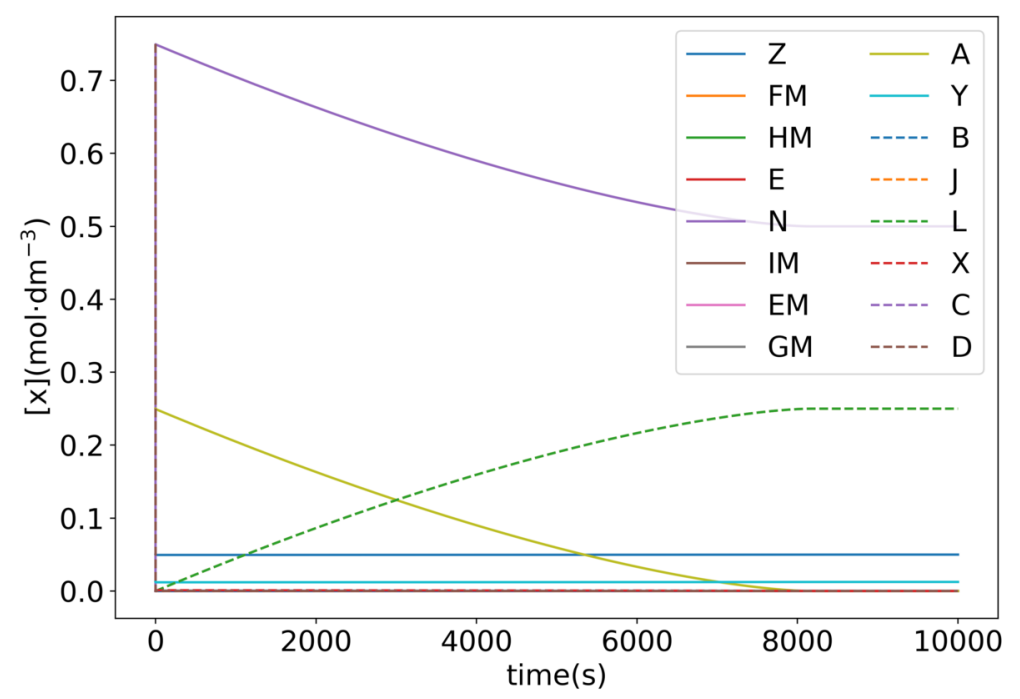

Figure 9: Concentration versus time graph of the favourable thermodynamic mechanism kinetic study. $\mathrm{N}$ : B2nep2: $\mathrm{NaOPh}$ adduct. L: fluoroborane ; A: fluorobenze; Z: phosphine; Y: Ni(COD)2. [A]0=0.25M; $[\mathrm{Z}] 0=0.05 \mathrm{M} ;[\mathrm{Y}] 0=0.0125 \mathrm{M} ;[\mathrm{C}] 0=0.75 ;[\mathrm{D}] 0=0.75$. 


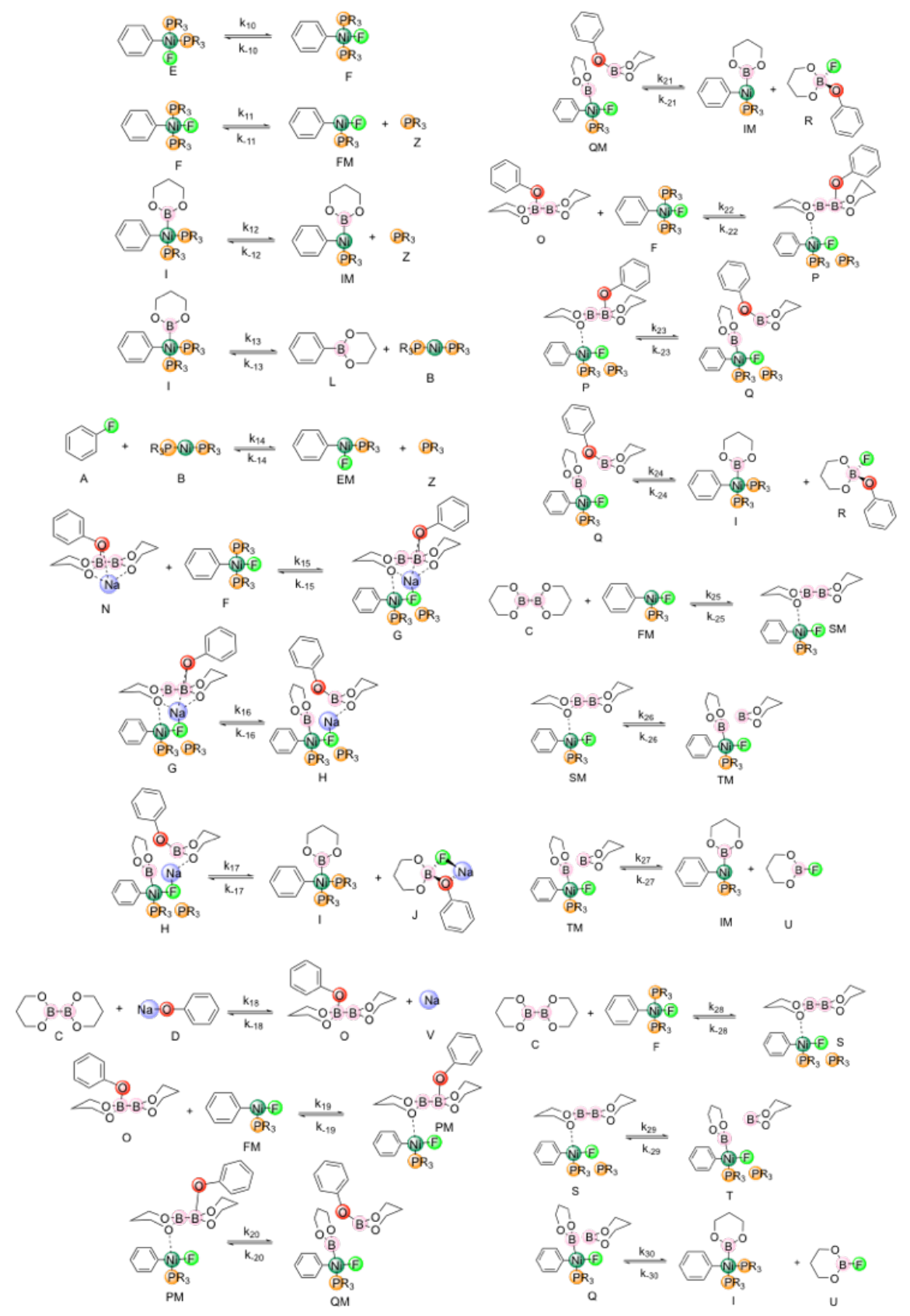

Figure 10: Additional elemental reactions

\section{Conclusions}

The mechanism of the borylation reaction of aryl fluoride assisted by a nickel catalyst and base was unravelled by means of DFT based methods. The presence of the sodium phenolate activates the diboron and modifies the classic cross-coupling mechanism. The reaction starts with the oxidative addition, where the metal catalyst breaks the C-F bond, followed by a cis/trans isomerization. This isomerization is more favourable for the mono-phosphine nickel compounds because steric effects. Then the trans intermediate reacts with 
the adduct $\mathrm{B}_{2}$ nep $_{2} / \mathrm{NaOPh}$ forming an stable pre-transmetalation species, which evolves to the first product of this reaction and an intermediate. This last one complex finally undergoes the reductive elimination, generating products and recovering the active catalytic species.

Kinetic modelling of the catalytic cycle taking into account the species involved in the most favourable mechanism only was not enough for reproducing the experimentally reported yield. Additional "off-cycle" equilibria, such as those bis-phosphine/mono-phosphine exchange reactions, showed key role in the overall kinetics.

ioChem-BD tools provided straightforward integration of data into this manuscript. 3D structures were very easily embedded by using the HTML code generated with just "on-click" procedure. The ioChemBD Reaction Energy Profile Report tool, generated using the plot.ly library, is just another piece aimed at enhancing the accuracy of our current computational catalyst discovery toolbox.

\section{References}

M. Álvarez-Moreno, C. de Graaf, N. López, F. Maseras, J. M. Poblet, and C. Bo. Managing the Computational Chemistry Big Data Problem: The ioChem-BD Platform. Journal of Chemical Information and Modeling, 55(1):95-103, dec 2014. doi: 10.1021/ci500593j. URL https://doi.org/10.1021\% 2Fci500593j.

Maria Besora and Feliu Maseras. Microkinetic modeling in homogeneous catalysis. Wiley Interdisciplinary Reviews: Computational Molecular Science, 8(6):e1372, jun 2018. doi: 10.1002/wcms.1372. URL https: //doi.org/10.1002\%2Fwcms. 1372.

C. Bo, F. Maseras, and N. López. The role of computational results databases in accelerating the discovery of catalysts. Nature Catalysis, 1:809-810, 2018.

Jeng-Da Chai and Martin Head-Gordon. Long-range corrected hybrid density functionals with damped atom-atom dispersion corrections. Physical Chemistry Chemical Physics, 10(44):6615, 2008. doi: 10. 1039/b810189b. URL https://doi.org/10.1039\%2Fb810189b.

Henry Eyring. The Activated Complex in Chemical Reactions. The Journal of Chemical Physics, 3(2): 107-115, feb 1935. doi: 10.1063/1.1749604. URL https://doi.org/10.1063\%2F1.1749604.

M. J. Frisch, G. W. Trucks, H. B. Schlegel, G. E. Scuseria, M. A. Robb, J. R. Cheeseman, G. Scalmani, V. Barone, G. A. Petersson, H. Nakatsuji, X. Li, M. Caricato, J. Bloino A. V. Marenich, B. G. Janesko, R. Gomperts, B. Mennucci, H. P. Hratchian, J. V. Ortiz, A. F. Izmaylov, J. L. Sonnenberg, D. WilliamsYoung, F. Ding, F. Lipparini, F. Egidi, J. Goings, B. Peng, A. Petrone, T. Henderson, D. Ranasinghe, V. G. Zakrzewski, J. Gao, N. Rega, G. Zheng, W. Liang, M. Hada, M. Ehara, K. Toyota, R. Fukuda, J. Hasegawa, M. Ishida, T. Nakajima, Y. Honda, O. Kitao, H. Nakai, K. Throssell T. Vreven, J. Montgomery, J. E. Peralta, F. Ogliaro, M. J. Bearpark, J. J. Heyd, K. N. Kudin E. N. Brothers, V. N. Staroverov, T. A. Keith, R. Kobayashi, J. Normand, K. Raghavachari, A. P. Rendell, J. C. Burant, S. S. Iyengar, J. Tomasi, M. Cossi, J. M. Millam, M. Klene, C. Adamo, R. Cammi, J. W. Ochterski, R. L. Martin, K. Morokuma, O. Farkas, J. B. Foresman, and 2016. D. J. Fox. Gaussian 09, Revision D.01.

Yeosan Lee, Seung yeol Baek, Jinyoung Park, Seoung-Tae Kim, Samat Tussupbayev, Jeongho Kim, MuHyun Baik, and Seung Hwan Cho. Chemoselective Coupling of 1,1-Bis[(pinacolato)boryl]alkanes for the Transition-Metal-Free Borylation of Aryl and Vinyl Halides: A Combined Experimental and Theoretical Investigation. Journal of the American Chemical Society, 139(2):976-984, dec 2016. doi: 10.1021/jacs. 6b11757. URL https://doi.org/10.1021\%2Fjacs.6b11757.

Soobin Lim, Dalnim Song, Seungwon Jeon, Youngsuk Kim, Hyunseok Kim, Sanghee Lee, Hyungdo Cho, Byung Chul Lee, Sang Eun Kim, Kimoon Kim, and Eunsung Lee. Cobalt-Catalyzed C-F Bond Borylation 
of Aryl Fluorides. Organic Letters, 20(22):7249-7252, nov 2018. doi: 10.1021/acs.orglett.8b03167. URL https://doi.org/10.1021\%2Facs .orglett.8b03167.

Xiang-Wei Liu, Javier Echavarren, Cayetana Zarate, and Ruben Martin. Ni-Catalyzed Borylation of Aryl Fluorides via C-F Cleavage. Journal of the American Chemical Society, 137(39):12470-12473, sep 2015. doi: 10.1021/jacs.5b08103. URL https://doi.org/10.1021\%2Fjacs. 5b08103.

Aleksandr V. Marenich, Christopher J. Cramer, and Donald G. Truhlar. Universal Solvation Model Based on Solute Electron Density and on a Continuum Model of the Solvent Defined by the Bulk Dielectric Constant and Atomic Surface Tensions. The Journal of Physical Chemistry B, 113(18):6378-6396, may 2009. doi: 10.1021/jp810292n. URL https://doi.org/10.1021\%2Fjp810292n.

Richard L. Martin, P. Jeffrey Hay, and Lawrence R. Pratt. Hydrolysis of Ferric Ion in Water and Conformational Equilibrium. The Journal of Physical Chemistry A, 102(20):3565-3573, may 1998. doi: 10.1021/jp980229p. URL https://doi.org/10.1021\%2Fjp980229p.

Takashi Niwa, Hidenori Ochiai, Yasuyoshi Watanabe, and Takamitsu Hosoya. Ni/Cu-Catalyzed Defluoroborylation of Fluoroarenes for Diverse C-F Bond Functionalizations. Journal of the American Chemical Society, 137(45):14313-14318, nov 2015. doi: 10.1021/jacs.5b10119. URL https://doi.org/10.1021\% 2Fjacs.5b10119.

Takashi Niwa, Hidenori Ochiai, and Takamitsu Hosoya. Copper-Catalyzed ipso-Borylation of Fluoroarenes. ACS Catalysis, 7(7):4535-4541, jun 2017. doi: 10.1021/acscatal.7b01448. URL https://doi.org/10. $1021 \% 2$ Facscatal. $7 \mathrm{~b} 01448$.

Hayate Saito, Shinya Otsuka, Keisuke Nogi, and Hideki Yorimitsu. Nickel-Catalyzed Boron Insertion into the C2-O Bond of Benzofurans. Journal of the American Chemical Society, 138(47):15315-15318, nov 2016. doi: 10.1021/jacs.6b10255. URL https://doi.org/10.1021\%2Fjacs.6b10255.

Hironobu Sakaguchi, Yuta Uetake, Masato Ohashi, Takashi Niwa, Sensuke Ogoshi, and Takamitsu Hosoya. Copper-Catalyzed Regioselective Monodefluoroborylation of Polyfluoroalkenes en Route to Diverse Fluoroalkenes. Journal of the American Chemical Society, 139(36):12855-12862, sep 2017. doi: 10.1021/jacs. 7b08343. URL https://doi.org/10.1021\%2Fjacs.7b08343.

Karen L. Schuchardt, Brett T. Didier, Todd Elsethagen, Lisong Sun, Vidhya Gurumoorthi, Jared Chase, Jun Li, and Theresa L. Windus. Basis Set Exchange: A Community Database for Computational Sciences. Journal of Chemical Information and Modeling, 47(3):1045-1052, apr 2007. doi: 10.1021/ci600510j. URL https://doi.org/10.1021\%2Fci600510j.

Martin C. Schwarzer, Ryosuke Konno, Takayuki Hojo, Akimichi Ohtsuki, Keisuke Nakamura, Ayaka Yasutome, Hiroaki Takahashi, Toshiaki Shimasaki, Mamoru Tobisu, Naoto Chatani, and Seiji Mori. Combined Theoretical and Experimental Studies of Nickel-Catalyzed Cross-Coupling of Methoxyarenes with Arylboronic Esters via C-O Bond Cleavage. Journal of the American Chemical Society, 139(30):10347-10358, jul 2017. doi: 10.1021/jacs.7b04279. URL https://doi.org/10.1021\%2Fjacs.7b04279.

Zhang-Jie Shi. Homogeneous Catalysis for Unreactive Bond Activation. John Wiley \& Sons Inc., sep 2014. doi: 10.1002/9781118788981. URL https://doi.org/10.1002\%2F9781118788981.

Henry Taube. Rates and Mechanisms of Substitution in Inorganic Complexes in Solution. Chemical Reviews, 50(1):69-126, feb 1952. doi: 10.1021/cr60155a003. URL https://doi.org/10.1021\%2Fcr60155a003.

Jiang Wang, María Sánchez-Roselló, José Luis Aceña, Carlos del Pozo, Alexander E. Sorochinsky, Santos Fustero, Vadim A. Soloshonok, and Hong Liu. Fluorine in Pharmaceutical Industry: Fluorine-Containing Drugs Introduced to the Market in the Last Decade (2001-2011). Chemical Reviews, 114(4):2432-2506, dec 2013. doi: 10.1021/cr4002879. URL https://doi.org/10.1021\%2Fcr4002879. 
Alexander Zapf. Homogeneous Catalysis. Understanding the Art. Von Piet W. N. M. van Leeuwen. Angewandte Chemie, 117(9):1321-1321, feb 2005. doi: 10.1002/ange.200485259. URL https://doi.org/10. $1002 \% 2$ Fange. 200485259.

Juan Zhang, Wenpeng Dai, Qingyun Liu, and Song Cao. Cu-Catalyzed Stereoselective Borylation of gemDifluoroalkenes with B2pin2. Organic Letters, 19(12):3283-3286, jun 2017. doi: 10.1021/acs.orglett. 7b01430. URL https://doi.org/10.1021\%2Facs.orglett.7b01430.

Jing Zhou, Maximilian W. Kuntze-Fechner, Rüdiger Bertermann, Ursula S. D. Paul, Johannes H. J. Berthel, Alexandra Friedrich, Zhenting Du, Todd B. Marder, and Udo Radius. Preparing (Multi)Fluoroarenes as Building Blocks for Synthesis: Nickel-Catalyzed Borylation of Polyfluoroarenes via C-F Bond Cleavage. Journal of the American Chemical Society, 138(16):5250-5253, apr 2016. doi: 10.1021/jacs.6b02337. URL https://doi.org/10.1021\%2Fjacs.6b02337. 